\title{
How to Deal with the Past Memories of Patients in Palliative Care: A Suggested New Approach

\author{
Eun-Seung Yu, Ph.D.
} \\ Department of Counseling Psychology, The Cyber University of Korea, Seoul, Korea
}

\begin{abstract}
Dealing with existential concerns experienced by patients is an important part of palliative care. Interventions that use the life review method to encourage patients to reminisce about their lives can help them find new positive meanings, promote ego integrity, and reduce emotional suffering. Not everyone has positive memories when they look back on the past, however. This poses a limit on the effectiveness of the life review method for healthcare providers working in palliative care contexts. In this study, we discuss the limits of life review and suggest imagery rescripting as a new modality constituting a psychotherapeutic approach to deal with negative memories safely and effectively.
\end{abstract}

Key Words: Historical trauma, Psychotherapy, Palliative care, Hospice care

\author{
Received May 20, 2021 \\ Revised May 21, 2021 \\ Accepted May 21, 2021 \\ Correspondence to \\ Eun-Seung Yu \\ ORCID: \\ https://orcid.org/0000-0001-7388-9195 \\ E-mail: psyesyu@gmail.com
}

\section{INTRODUCTION}

It is inevitable for patients with advanced diseases to experience complex physical symptoms and psychological issues. A number of studies have emphasized the importance of dealing with existential concerns faced by patients at the end of their lives for their psychological adaptation [1,2]. It was recently found that various psychotherapeutic approaches for existential issues such as meaning-centered psychotherapy, dignity therapy, and Managing Cancer and Living Meaningfully (CALM) therapy decreased psychological distress and improved spiritual well-being in patients who received palliative and hospice care. These therapeutic interventions represent a supportive-expressive approach, and they commonly involve life review, in which patients share stories about their life and past. At the end of life, people tend to return to their past $\mathrm{ex}^{-}$ periences and reflect on unresolved issues and consequent regrets, which in turn promotes a sense of self-integration. This is because expressing and interpreting one's feelings about the past through dialogue can help one to accept the present self positively and to naturally accept and prepare the mind for death. In this sense, a life review, in which one recalls his or her life and past, gives patients the opportunity to identify and reflect on past experiences and achievements to find meaning and address unresolved issues. As such, life review is considered another form of existential therapy [3].

In this study, the life review approach using patients' life logs in the context of palliative and hospice care was reviewed, and its limitations in the course of dealing with negative past experiences was discussed. Imagery rescripting, which is a type of psychotherapeutic approach, is suggested as a novel modality and complementary method for overcoming this limitation.

\section{LIFE REVIEW APPROACH}

\section{Life review and reminiscence}

According to Erikson's development theory, a person's fi-

This is an Open Access article distributed under the terms of the Creative Commons Attribution Non-Commercial License (http://creativecommons.org/licenses/by-nc/4.0/) which permits unrestricted non-commercial use, distribution, and reproduction in any medium, provided the original work is properly cited. 
nal developmental task is to achieve ego integrity through a process of reinterpreting and accepting one's past life [4]. For patients with advanced life-threatening medical illnesses, it may also be important for them to recall their past good and bad experiences and integrate those into their understanding of their lives by discovering new meanings in the context of their life trajectories.

A life review approach in which elderly people recall meaningful past experiences is known to be effective for psychological adaptation and ego integration $[5,6]$. In the life review approach, reminiscence is considered an effective skill for helping individuals later in their lives [7]. Reminiscence is defined as the act or process of recalling past events or experiences [8]. It is a simple tool that can mitigate potentially devastating emotional problems and promote the psychological health of individuals. The value of reminiscence in hospice care has been emphasized by many researchers [8].

\section{Life review approach in palliative care}

In a palliative care setting, life review commonly consists of six 1-hour sessions: an interview in chronological order, a summary, an evaluation, and a reconstruction of one's life [9]. It can be either individual or group-based. In life review therapy, a patient is asked specific questions about his or her life and instructed to spend some time reminiscing on various past experiences [10]. Wholihan [8] emphasized the therapeutic value of reminiscence and suggested a series of questions to facilitate the process of reminiscence in hospice care by applying Butler's work on the life review process [5]. Possible questions pertain to one's past work, one's accomplishments, one' s happiest day, one's happy childhood memories, particular holidays that hold special meaning, the 'first' memories, one' s relationship with a best friend, times when the person helped someone, and particular places that hold special meaning. When patients review their life and past, it is not sufficient for them to simply recall their life. When psychological counseling techniques such as acceptance, encouragement, and storytelling are used in conjunction with reminiscence so that psychological conflicts that appear during life review can be resolved and the meaning of one's life can be reconstructed, the therapeutic effect becomes stronger [11]. Therefore, all healthcare personnel are potential guides for reminiscence and should receive formal education regarding life review therapy [8].

Dignity therapy, meaning-centered therapy, and CALM therapy deal with existential issues and spiritual suffering in great depth in palliative and hospice care settings, and they also include life review as a main component. While life review therapy can be conducted at the bedside via daily interviews, in-depth psychotherapeutic interventions such as dignity therapy represent a more formal approach to life review [12]. Reminiscence can have various adaptive functions, such as developing a retrospective view of one's life and mediating past conflicts throughout the intervention [5].

Psychological intervention using life review is known to decrease emotional distress, including depression and anxiety, as well as increase the sense of meaning in life among patients in palliative care settings $[13,14]$. Life review can be a relatively safe and effective means for patients with life-threatening illnesses to integrate their past memories into their larger life story, even for traumatic memories [15]. However, there is a potential risk for re-traumatization if a patient has intense traumatic memories, with some life reviews leading to negative emotional outcomes [16]. Kunz and Soltys [15] concluded that opening up painful memories associated with traumatic events during life review needs to be managed appropriately in order for healing to occur. Therefore, a complementary approach is needed so that psychological approaches using life review can safely and effectively deal with patient's past negative and traumatic experiences.

\section{TRAUMATIC MEMORIES}

\section{Traumatic memories in human beings}

Not everyone feels positive emotions when they look back on the past. Up to $84 \%$ of individuals experience a traumatic event during their lives, making trauma an almost universal experience [17]. One study showed that cancer survivors experienced post-traumatic stress disorder (PTSD) about 1.66 times more often than the general population, and that many of those traumatic events were unrelated to cancer [18].

The intrusive memories of patients with PTSD usually directly involve their struggles with illness. Many cases of PTSD at the end of life originate from past trauma, since traumatic 
memories can be reactivated at the end of life [19]. Even when patients do not have PTSD, inner conflicts caused by negative, sometimes traumatic, experiences can persist for years or even an entire lifetime. Such inner conflicts can essentially end or be forgotten once they are resolved or overcome [20]. Intense, unresolved inner conflicts contribute greatly to emotional suffering and cause anxiety. For people who have faced hardships in their lives, regrets and conflicts often become apparent at the end of life. It is more important to address negative memories than positive memories to achieve integration of one's memories and have a successful life review [6]. Moreover, if a patient experienced a traumatic event and one's emotional suffering and pain resulting from that memory are enough to affect the patient's life at the present moment, it is very important to take a therapeutic approach to address these issues. Psychological conflicts can be resolved during treatment for overcoming traumatic experiences, and one's sense of the meaning of his or her life can be reconstructed using this approach. In one study, it was found that patients who experienced a successful life review showed a significantly higher degree of integration and instrumental reminiscence and less obsessive reminiscence than patients with unsuccessful life reviews [21]. Therefore, an in-depth approach to address patients' negative, traumatic experiences can be an important factor for improving the effectiveness of life review therapy in palliative care settings.

\section{Dealing with traumatic memories in palliative and hospice care}

Traditional evidence-based treatments for PTSD, such as prolonged exposure, cognitive processing therapy, and eye movement desensitization and reprocessing, mostly require eight to 16 weekly sessions [22]. In contrast to the typical duration of treatment for PTSD, however, the median duration of a hospice stay in South Korea is 19.1 days [23], which is longer than the median duration is in the U.S. (17.4 days) [24], but still too short to conduct full treatment for PTSD. Many patients may pass away before therapy for PTSD is complete. In addition, the general condition of administered patients is often poor, so it can be challenging for patients to participate in lengthy sessions that typically last 50 to 90 minutes. In addition, patients receiving traditional PTSD treatment tend to paradoxically experience an increase in PTSD symptoms early in treatment, usually until around the sixth session [25]. For these reasons, it can be difficult to conduct traditional intervention for PTSD that arises during end of life care. Therefore, it is necessary to take a safe approach to address the traumatic memories of patients suffering illnesses at the end of life.

\section{IMAGERY RESCRIPTING (IR) AS A PSYCHOTHERAPY}

Psychotherapists cannot alter the past. However, they can help patients to reinterpret past events that trouble them and to attenuate the real-life impact of negative memories. Imagery rescripting (IR), which is an imagery-focused treatment developed by Smucker, aims to assist patients in reinterpreting negative memories via the imagination and lead them to a "more favorable ending" using various techniques derived from an introductory example $[26,27]$. Due to their nature, traumatic memories are more likely to be encoded in memory in visual or sensorimotor modalities rather than verbal ones. In other words, early traumatic memories are encoded primarily in images. Therefore, it is suggested that the use of imagery is essential in transforming the meaning of traumatic memories [26].

IR is conducted in two phases, beginning with exposure and ending with a rescripting procedure [26]. The patient is instructed to imagine the memory or image as vividly as possible, as if it were really happening to him or her at that present moment (exposure phase). Next, the patient is instructed to imagine the sequence of events being changed according to his or her desired outcomes (rescripting phase) [28]. In this rescripting phase, the therapist asks the patient to rescript the traumatic scene into mastery imagery (i.e., by imagining what the adult self would do to change the course of events according to the past self s desires) and adult-nurturing-child imagery (i.e., by imagining nurturing the past self by changing the course of events according to the adult self's desires). Essentially, the role of the current self during rescripting is to rescue and protect the past self from any further traumatic events [26]. Through IR, patients can develop a sense of control after imagining mastery imagery, thereby enhancing their sense of self-nurturing and self-soothing. Patients can reattribute and change the meaning of the traumatic event and also feel that 
they are being taken care of, even in the imagination. This process has a powerful therapeutic effect [28]. In addition, IR can be beneficial since it is less likely to retraumatize patients and can be applied to a wider range of problems than exposure-based therapy [28].

This series of IR procedures can be applied to cancer patients exactly as described. There have been attempts to conduct IR with cancer patients, although not in cases of advanced cancer, and studies have reported that IR may be appropriate for cancer patients who experience intrusive memories, anxiety, and depression [29,30]. In particular, one case study found that patients felt as if they could regain control over the situation using mastery imagery following a cancer diagnosis and reduce feelings of guilt, worthlessness, and hopelessness using compassionate imagery. These effects were relatively longlasting, as observed at a6-month follow-up appointment [30]. Thus, IR may be effective at transforming patients' painful memories and images into positive images, rather than just reminiscing about the past. Therefore, when clinicians conduct life review therapy for cancer patients at the end of their lives, the IR technique can be helpful for freeing patients from painful memories of the past and integrating traumatic experiences as a part of one's life, helping patients to develop a sense of meaning about their lives, and enabling patients to experience the present moment.

\section{CONCLUSION}

Dealing with existential concerns faced by patients is an important part of palliative care. Interventions using life review therapy in which patients reminisce about their pasts can lead to finding new positive meanings, promote ego integrity, and reduce emotional suffering. In the course of addressing existential concerns, it is important for patients to find happiness and meaning when reviewing their lives, but dealing with the negative emotions and lingering conflicts uncovered by the life review process can be even more meaningful and important. However, there is a potential risk for re-traumatization if $\mathrm{pa}^{-}$ tients have intense traumatic memories. Furthermore, given that trauma is considered an almost universal experience in any person's life trajectory, clinicians who provide palliative and hospice care need to know how to deal effectively with negative and traumatic memories and patients' emotional responses to such memories during the life review process. IR is a psychotherapeutic technique for safely and effectively dealing with negative or traumatic memories. Using imagination, patients are guided by a therapist to replace negative images resulting from previous traumatic events with new imagesparticularly mastery imagery, but also compassionate and nurturing imagery. IR can be used for patients in palliative care settings who suffer from negative or traumatic memories to free them from unresolved emotions and help them find positive meanings in their lives. Clinicians who provide palliative care are encouraged to use this technique to help patients deal with previous negative experiences safely and effectively.

\section{CONFLICT OF INTEREST}

No potential conflict of interest relevant to this article was reported.

\section{REFERENCES}

1. Lee JH. Existential issues and psychosocial interventions in palliative care. Korean J Hosp Palliat Care 2020;23:167-71.

2. Breitbart W, Gibson C, Poppito SR, Berg A. Psychotherapeutic interventions at the end of life: a focus on meaning and spirituality. Can J Psychiatry 2004;49:366-72.

3. Grassi L, Riba M. Clinical psycho-oncology: an international perspective. Chichester:John Wiley \& Sons Ltd;2012.

4. Erikson EH. Childhood and society. New York:WW Norton;c1950.

5. Butler RN. The life review: an interpretation of reminiscence in the aged. Psychiatry 1963;26:65-76.

6. Haight BK, Bahr RT. The therapeutic role of the life review in the elderly. Academic Psychol Bull 1984;6:289-9.

7. Cappeliez P, Rivard V, Guindon S. Functions of reminiscence in later life: proposition of a model and applications. Eur Rev Appl Psychol 2007:57:151-6.

8. Wholihan D. The value of reminiscence in hospice care. Am J Hosp Palliat Care 1992;9:33-5. 
9. Haight BK. The therapeutic role of a structured life review process in homebound elderly subjects. J Gerontol 1988;43:P40-4.

10. Fitzgerald P, Nissim R, Rodin G. A life-stage approach to psycho-oncology. Clinical Psycho-Oncology 2012:155-63.

11. O’leary E, Barry N. Reminiscence therapy with older adults. J Soc Work Pract 1998;12:159-65.

12. Thompson GN, Chochinov HM. Dignity-based approaches in the care of terminally ill patients. Curr Opin Support Palliat Care 2008;2:49-53.

13. Ando M, Tsuda A, Morita T. Life review interviews on the spiritual well-being of terminally ill cancer patients. Support Care Cancer 2007:15:225-31.

14. Ahn SH, An YL, Yoo YS, Ando M, Yoon SJ. Effects of a short-term life review on spiritual well-being, depression, and anxiety in terminally ill cancer patients. J Korean Acad Nurs 2012;42:28-35.

15. Kunz JA, Soltys FG. Transformational reminiscence : life story work. New York:Springer Publishing Company;2007.

16. Westwood MJ, McLean HB. Traumatic memories and life review. In: Kunz JA, Soltys FG, eds. Transformational reminiscence: Life story work. New York:Springer Publishing Company;2007. p. 181-96.

17. Benjet C, Bromet E, Karam EG, Kessler RC, McLaughlin KA, Ruscio AM, et al. The epidemiology of traumatic event exposure worldwide: results from the World Mental Health Survey Consortium. Psychol Med 2016;46:327-43.

18. Swartzman S, Booth JN, Munro A, Sani F. Posttraumatic stress disorder after cancer diagnosis in adults: A meta analysis. Depress Anxiety 2017;34:327-39.

19. Wilson KG, Lander M, Chochinov HM. Diagnosis and management of depression in palliative care. In: Chochinov HM, Breitbart W. Handbook of psychiatry in palliative medicine. 2nd ed. Oxford:Oxford University Press;2000. p. 39-68.

20. McReynolds P. The nature and logic of intrapsychic conflicts. In: Spielberger CD, Sarason IG, Strelau J, Brebner JMT, eds. he series in clinical psychology and The series in clinical and community psychology. Stress and anxiety, Vol. 13. New York;London:Hemisphere;1991. p. $73-83$.

21. Wong PT, Watt LM. What types of reminiscence are associated with successful aging? Psychol Aging 1991;6:272-9.

22. Feldman DB. Stepwise Psychosocial Palliative Care: A New Approach to the Treatment of Posttraumatic Stress Disorder at the End of Life. J Soc Work End Life Palliat Care 2017;13:113-33.

23. Lee JE, Goo AJ, Cho BL. The current status of end-of-life care in Korea and legislation of well-dying act. AGMR 2016;20:65-70.

24. NHPCO. NHPCO's facts and figures : hospice care in America 2015. Alexandria, VA:National Hospice and Palliative Care Oranization;2015.

25. Nishith P, Resick PA, Griffin MG. Pattern of change in prolonged exposure and cognitive-processing therapy for female rape victims with posttraumatic stress disorder. J Consult Clin Psychol 2002;70:880-6.

26. Smucker MR, Dancu C, Foa EB, Niederee JL. Imagery rescripting: A new treatment for survivors of childhood sexual abuse suffering from posttraumatic stress. J Cogn Psychother 1995;9:3-17.

27. Moritz S, Ahlf-Schumacher J, Hottenrott B, Peter U, Franck S, Schnell T, et al. We cannot change the past, but we can change its meaning. A randomized controlled trial on the effects of self-help imagery rescripting on depression. Behav Res Ther 2018;104:74-83.

28. Arntz A. Imagery rescripting as a therapeutic technique: Review of clinical trials, basic studies, and research agenda. J Exp Psychopathol 2012;3:189-208.

29. Sadeghi E, Ghorbanshiroudi S, Tarkhan M, Keyhanian S. The Effect of imagery rescripting and reprocessing therapy on negative automatic thoughts and dysfunctional attitudes of women with cancer. Soc Cogn 2018;6:155-66.

30. Whitaker KL, Brewin CR, Watson M. Imagery rescripting for psychological disorder following cancer: a case study. Br J Health Psychol 2010;15(Pt 1):41-50. 